\title{
Uniform nanoparticles by flame- assisted spray pyrolysis (FASP) of low cost precursors
}

\section{Journal Article}

Author(s):

Rudin, Thomas; Wegner, Karsten; Pratsinis, Sotiris E.

Publication date:

2011-07

Permanent link:

https://doi.org/10.3929/ethz-b-000037456

Rights / license:

In Copyright - Non-Commercial Use Permitted

Originally published in:

Journal of Nanoparticle Research 13(7), https://doi.org/10.1007/s11051-010-0206-x 


\title{
Uniform nanoparticles by flame-assisted spray pyrolysis (FASP) of low cost precursors
}

\author{
Thomas Rudin • Karsten Wegner • \\ Sotiris E. Pratsinis
}

Received: 21 June 2010/Accepted: 21 December 2010/Published online: 12 January 2011

(C) Springer Science+Business Media B.V. 2011

\begin{abstract}
A new flame-assisted spray pyrolysis (FASP) reactor design is presented, which allows the use of inexpensive precursors and solvents (e.g., ethanol) for synthesis of nanoparticles (10-20 nm) with uniform characteristics. In this reactor design, a gas-assisted atomizer generates the precursor solution spray that is mixed and combusted with externally fed inexpensive fuel gases (acetylene or methane) at a defined height above the atomizing nozzle. The gaseous fuel feed can be varied to control the combustion enthalpy content of the flame and onset of particle formation. This way, the enthalpy density of the flame is decoupled from the precursor solution composition. Low enthalpy content precursor solutions are prone to synthesis of non-uniform particles (e.g., bimodal particle size distribution) by standard flame spray pyrolysis (FSP) processes. For example, metal nitrates in ethanol typically produce nanosized particles by gas-to-particle conversion along with larger particles by droplet-to-particle conversion. The present FASP design facilitates the use of such low enthalpy precursor solutions for synthesis of homogeneous nanopowders by increasing the combustion enthalpy density of the flame with low-cost, gaseous fuels. The effect of flame enthalpy density on product
\end{abstract}

T. Rudin $\cdot$ K. Wegner $\cdot$ S. E. Pratsinis $(\square)$ Department of Mechanical and Process Engineering, Particle Technology Laboratory, Institute of Process Engineering, ETH Zurich, 8092 Zurich, Switzerland e-mail: sotiris.pratsinis@ptl.mavt.ethz.ch properties in the FASP configuration is explored by the example of $\mathrm{Bi}_{2} \mathrm{O}_{3}$ nanoparticles produced from bismuth nitrate in ethanol. Product powders were characterized by nitrogen adsorption, X-ray diffraction, X-ray disk centrifuge, and transmission electron microscopy. Homogeneous $\mathrm{Bi}_{2} \mathrm{O}_{3}$ nanopowders were produced both by increasing the gaseous fuel content and, most notably, by cutting the air entrainment prior to ignition of the spray.

Keywords $\mathrm{Bi}_{2} \mathrm{O}_{3}$. Flame assisted spray pyrolysis . Flame spray pyrolysis - Gas phase synthesis . Low-cost production - Flame enthalpy density . Product homogeneity $\cdot$ Bimodal size distribution

\section{Introduction}

In recent years, combustion of aerosols of organic solutions, the so-called flame spray pyrolysis (FSP) process (Lewis 1991; Bickmore et al. 1996; Madler et al. 2002a), has been proven to be an efficient method for synthesis of single- and multi-component nanoparticles with tailored characteristics and high purity, that find many applications including catalysis (Strobel et al. 2006), gas sensors (Sahm et al. 2004), nutritional supplements (Rohner et al. 2007), and biomaterials (Loher et al. 2005). This is a scalable technology (Mueller et al. 2003) for synthesis of 
advanced materials ready to take the next step from the laboratory to industrial scale manufacturing (Strobel and Pratsinis 2007).

Toward that goal, reduction of production costs can be achieved using inexpensive precursors (e.g., nitrates or carbonates) and solvents (e.g., ethanol or water). Such precursor solutions, however, tend to form mixtures of nanoparticles made by gas-to-particle conversion and much larger, irregular (hollow or fragmented) particles by droplet-to-particle conversion (Madler et al. 2002b). For example, bismuth oxide produced by FSP of its nitrate precursor in ethanol and nitric acid, resulted in hollow $(1 \mu \mathrm{m}$ in diameter $)$ and compact $\mathrm{Bi}_{2} \mathrm{O}_{3}$ particles (50-100 nm in diameter) as well as aggregates of primary particles, each about $10 \mathrm{~nm}$ in diameter (Madler and Pratsinis 2002). In contrast, dissolution of bismuth nitrate in acetic acid led exclusively to synthesis of small, solid nanoparticles. Similarly, cerium acetate in pure acetic acid yielded non-uniform $\mathrm{CeO}_{2}$ particles while replacing it with iso-octane/2-butanol accelerated precursor evaporation and gas-phase reaction, resulting in uniform powders (Madler et al. 2002b). Also using inexpensive ethanol-based yttrium nitrate/zirconium propoxide precursor solutions for yttrium-stabilized zirconia resulted in non-uniform products, while dissolution of yttrium butoxide/zirconium propoxide precursors in toluene resulted in uniform nanoparticles (Jossen et al. 2005a).

For a number of products, relatively expensive, oxygenated liquid precursor solutions such as carboxylic acid-derived compounds facilitate synthesis of uniform nanomaterials (Stark et al. 2003). The production of non-uniform FSP products is avoided also by use of high enthalpy precursors and/or solvents that tend to be costly, however. On the other hand, low-cost precursors and solvents are used when the heat for pyrolysis of the spray solution is provided by a separate source, e.g., a hot-wall oven in conventional spray pyrolysis (Messing et al. 1993) or a hot gaseous flame in flame-assisted spray pyrolysis (FASP) (Marshall et al. 1971) making these processes less sensitive to the choice of precursors and solvents (Madler 2004). Typically in FASP, a spray of precursor solution is generated by a pressure-driven (Matsoukas and Friedlander 1991) or ultrasonic (Kilian and Morse 2001) atomizer and carried into a flame, which in contrast to FSP, provides most of the combustion enthalpy, driving precursor decomposition and product particle formation. For FASP, most combustion enthalpy comes from a gas-fed flame rather than the precursor solution as in standard FSP, where a gas flame is used only to ignite and sustain spray combustion by facilitating spray droplet evaporation.

FASP has been used to produce superconductors (Merkle et al. 1990), phosphors (Purwanto et al. 2008), electroceramics (Seo et al. 2006), and perovskites for catalysis (Kriegel et al. 1994) to name only a few functional materials. Also gas-assisted atomizers have been used to inject a spray of water or organic solvents into a hydrogen/oxygen flame (Tikkanen et al. 1997) for production of manganese oxide and alumina. Seo et al. (2003) produced $\mathrm{ZnO}, \mathrm{MgO}$, and $\mathrm{NiO}$ nanoparticles by FASP of aqueous nitrate and acetate precursor solutions in a propane/oxygen flame. For low propane flow rates and thus low flame temperatures, large hollow or dense particles were observed similar to non-uniform ones made by conventional FSP (Jossen et al. 2005b). When using high fuel flow rates, however, the formation of small nanoparticles was reported (Seo et al. 2003).

For production of uniform nanosized particles in FSP, it seems thus possible that the production cost might be reduced if part of the energy of the organic solvents was replaced by low cost gaseous fuels like hydrogen or hydrocarbons, comparable to FASP. This study explores particle formation by a novel FASP reactor configuration that allows decoupling the flame enthalpy content from the precursor chemistry in conventional FSP. This reactor enables changing the enthalpy density of the reaction zone by providing additional gaseous fuels or, more importantly, controlling the air entrainment (Heine et al. 2006) in the spray prior to its ignition without changing, however, the composition of the precursor solution or its delivery rate into the flame. The formation of uniform bismuth oxide nanopowders is demonstrated by FASP of precursors that produce non-uniform $\mathrm{Bi}_{2} \mathrm{O}_{3}$ particle size distributions by conventional FSP (Madler and Pratsinis 2002).

\section{Experimental}

Particle synthesis

Bismuth oxide nanoparticles were made by FSP as described by Madler and Pratsinis (2002). Bismuth 
trinitrate pentahydrate $\left(\mathrm{Bi}\left(\mathrm{NO}_{3}\right)_{3} \cdot 5 \mathrm{H}_{2} \mathrm{O}\right.$; purity $>98 \%$; Aldrich) was dissolved in 15 vol.\% concentrated nitric acid (65\% in water, Fluka) and denaturized absolute ethanol (A15, Alcosuisse) to give a Bi concentration of $0.4 \mathrm{~mol} / \mathrm{L}$. The solution was fed through the spray nozzle (capillary inner/outer diameter $0.41 / 0.72 \mathrm{~mm}$ ) at $2 \mathrm{~mL} / \mathrm{min}$ by a syringe pump (Lambda, VIT-FIT) and atomized by co-flowing $6 \mathrm{~L} / \mathrm{min}$ of oxygen $(\geq 99.5 \%$, PanGas) through a surrounding annulus $(0.97 \mathrm{~mm} \mathrm{o.d.)}$ at $1.5 \mathrm{bar}$ pressure drop. In addition, $5 \mathrm{~L} / \mathrm{min}$ (sheath) $\mathrm{O}_{2}$ was supplied through 32 holes of $0.8 \mathrm{~mm}$ diameter each surrounding the precursor capillary at a distance of $1.5 \mathrm{~cm}$. All gas flow rates were controlled by calibrated mass flow controllers (Bronkhorst, EL-FLOW). Using a vacuum pump (Busch, Mink MM1202 AV), product particles were collected on water-cooled glass microfiber filters (Whatman GF 6, $25.7 \mathrm{~cm}$ in diameter) placed at least $60 \mathrm{~cm}$ above the burner.

Two experimental configurations were investigated for the ignition/support flame: In the first, standard FSP configuration (Madler et al. 2002b), the precursor-oxygen spray was ignited by a circular premixed flame of $1.5 \mathrm{~L} / \mathrm{min} \mathrm{CH}_{4}(\geq 99.5 \%$, PanGas) and $2.5 \mathrm{~L} / \mathrm{min} \mathrm{O}_{2}$ that surrounds the nozzle at the burner face. In the second, the flame-assisted spray pyrolysis configuration, the ignition/supporting flame was issued from a cylindrical torus ring (hereafter called ring) at selected heights above the nozzle (HAN) (Fig. 1). The ring was made of stainless steel with $1.6 / 2.5 \mathrm{~cm}$ inner/outer diameter and a length or height of $0.85 \mathrm{~cm}$. A steel tube of $2 \mathrm{~mm}$ i.d. delivered gas to the outer ring side at half of its length. In the inner cylindrical ring side, twelve holes $0.5 \mathrm{~mm}$ in diameter each were located equidistantly along its circumference, $2 \mathrm{~mm}$ below its top. These holes pointed radially inward at an inclination of $45^{\circ}$ downstream to ensure rapid mixing with the spray jet. The ring was placed coaxially to the nozzle axis so the level of these holes was at $\mathrm{HAN}=1-3 \mathrm{~cm}$. Through these holes either $1.5 \mathrm{~L} / \mathrm{min} \mathrm{CH}_{4}$ premixed with $2.5 \mathrm{~L} / \mathrm{min} \mathrm{O}_{2}$ or $1-2.5 \mathrm{~L} / \mathrm{min}$ of pure $\mathrm{C}_{2} \mathrm{H}_{2}(\geq 99.5 \%$, PanGas) were fed. Attention was paid that no spray droplets bypassed or hit the ring. A stainless steel tube (i.d. $1.6 \mathrm{~cm}$; o.d. $1.8 \mathrm{~cm}$ ) of variable length could be placed between the burner face and the ring to shield the spray from air entrainment (Fig. 1).

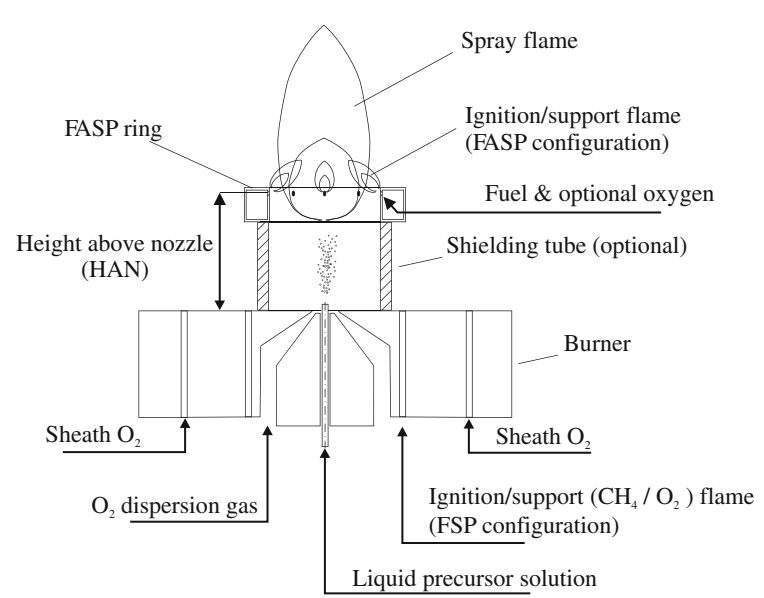

Fig. 1 Flame aerosol reactor with two-phase nozzle for atomization of the liquid precursor solution and cylindrical torus ring for injection of $\mathrm{C}_{2} \mathrm{H}_{2}$ or premixed $\mathrm{CH}_{4} / \mathrm{O}_{2}$ in flameassisted spray pyrolysis (FASP) configuration. The ring can be placed at various heights above the nozzle (HAN) of $0.5-3 \mathrm{~cm}$. Optionally, the spray could be surrounded by a shielding tube placed between the burner face and the FASP ring to prevent air entrainment

\section{Particle characterization}

The specific surface area (SSA) of the nanopowders was determined by $\mathrm{N}_{2}$ adsorption at $77 \mathrm{~K}$ (Tristar, Micromeritics Inc.) employing the BET isotherm. Assuming all particles to be monodispersed spheres, the BET-equivalent particle diameter was calculated as $\mathrm{d}_{\mathrm{BET}}=6$ / $\left(\rho_{\mathrm{Bi} 2 \mathrm{O} 3} \times \mathrm{SSA}\right)$, with $\rho_{\mathrm{Bi} 2 \mathrm{O} 3}=8900 \mathrm{~kg} / \mathrm{m}^{3}$. The particle morphology was obtained by transmission electron microscopy (TEM, Philips CM30). High-resolution transmission electron microscopy (HRTEM, FEI TECNAI F30, field-emission cathode operated at $300 \mathrm{kV}$ ) was employed to further characterize particle morphology and crystallinity. Particle size distributions were measured with an X-ray disc centrifuge (Brookhaven Instruments, BI XDC) at 1,500 rpm for $5 \mathrm{~h}$ (Schulz et al. 2008) after the agglomerated product powders were dispersed in deionized water by 5 min ultrasonication (Vibracell VCX 600, Sonics \& Materials Inc. $600 \mathrm{~W}, 20 \mathrm{kHz}, 13 \mathrm{~mm}$ tip, $0.5 \mathrm{~s}$ pulse, $0.3 \mathrm{~s}$ pause, $70 \%$ amplitude) forming $0.6 \mathrm{wt} \%$ suspensions. Particle suspensions showed sufficient stability to be analyzed as prepared. After a few days, however, sedimentation could be observed.

Crystallite sizes were determined by X-ray diffraction (XRD; Bruker D8 Advance, 40 kV, 40 mA, Cu K $\alpha$ radiation, Bragg-Brentano geometry) over a range of 
$20^{\circ}<2 \theta<60^{\circ}$ with a step size of $0.05^{\circ}$. The XRD spectra were analyzed using Topas 2.0 software (Bruker AXS, 2000). Phase information and crystal sizes were obtained by Rietveld refinement (Cheary and Coelho 1992) based on the crystalline data of $\mathrm{Bi}_{2} \mathrm{O}_{3}$ (Blower and Greaves 1988). The full XRD spectrum was fitted assuming Lorentzian peak shapes. Micro-strain was not considered. For products with broad or bimodal crystallite size distribution, the $\mathrm{Bi}_{2} \mathrm{O}_{3}$ pattern was reconstructed by two superimposed modes using a narrow and a wide Lorentzian with a large $(200 \mathrm{~nm})$ and small $(20 \mathrm{~nm})$ initial size for the Rietveld refinement (Madler and Pratsinis 2002). Thereby the average crystallite sizes and mass-fractions for each mode were obtained. A linear function was used for background correction, as the $\mathrm{Bi}_{2} \mathrm{O}_{3}$ spectra were well resolved to the baseline. Error bars represent the variation between different powder samples made at same conditions. To estimate the overall quality of the reconstructed XRD pattern, the "goodness of fit" (GOF) figure of merit was used (McCusker et al. 1999). The GOF-value is an index of agreement between calculated and measured pattern. Such values close to one represent a better refinement of the crystal structure (McCusker et al. 1999).

\section{Results and discussion}

\section{Flame structure}

Figure 2a shows a spray flame producing $\mathrm{Bi}_{2} \mathrm{O}_{3}$ nanoparticles with $2.5 \mathrm{~L} / \mathrm{min} \mathrm{C}_{2} \mathrm{H}_{2}$ and the FASP ring at $\mathrm{HAN}=1 \mathrm{~cm}$. The flame is burning stable above the ring while no flame is observed between the spray nozzle and the ring. The visible flame length was approximately $5 \mathrm{~cm}$ above the ring. When the ring was placed at $\mathrm{HAN}=3 \mathrm{~cm}$, a wide flame was formed with a crosssection about the size of the ring (Fig. 2b). The visible flame length was slightly shorter, approximately $4 \mathrm{~cm}$. Only a small change in visible flame length was observed when the feed of the $\mathrm{Bi}_{2} \mathrm{O}_{3}$ precursor solution was stopped, resulting in a pure acetylene diffusion flame. At this flow rate, the acetylene stream provided three times as much combustion enthalpy as the precursor solution. The spray flames were burning stable up to $\mathrm{HAN}=3 \mathrm{~cm}$. Larger HAN were not investigated as the non-burning precursor spray widened and formed particle deposits on the inner ring surface.
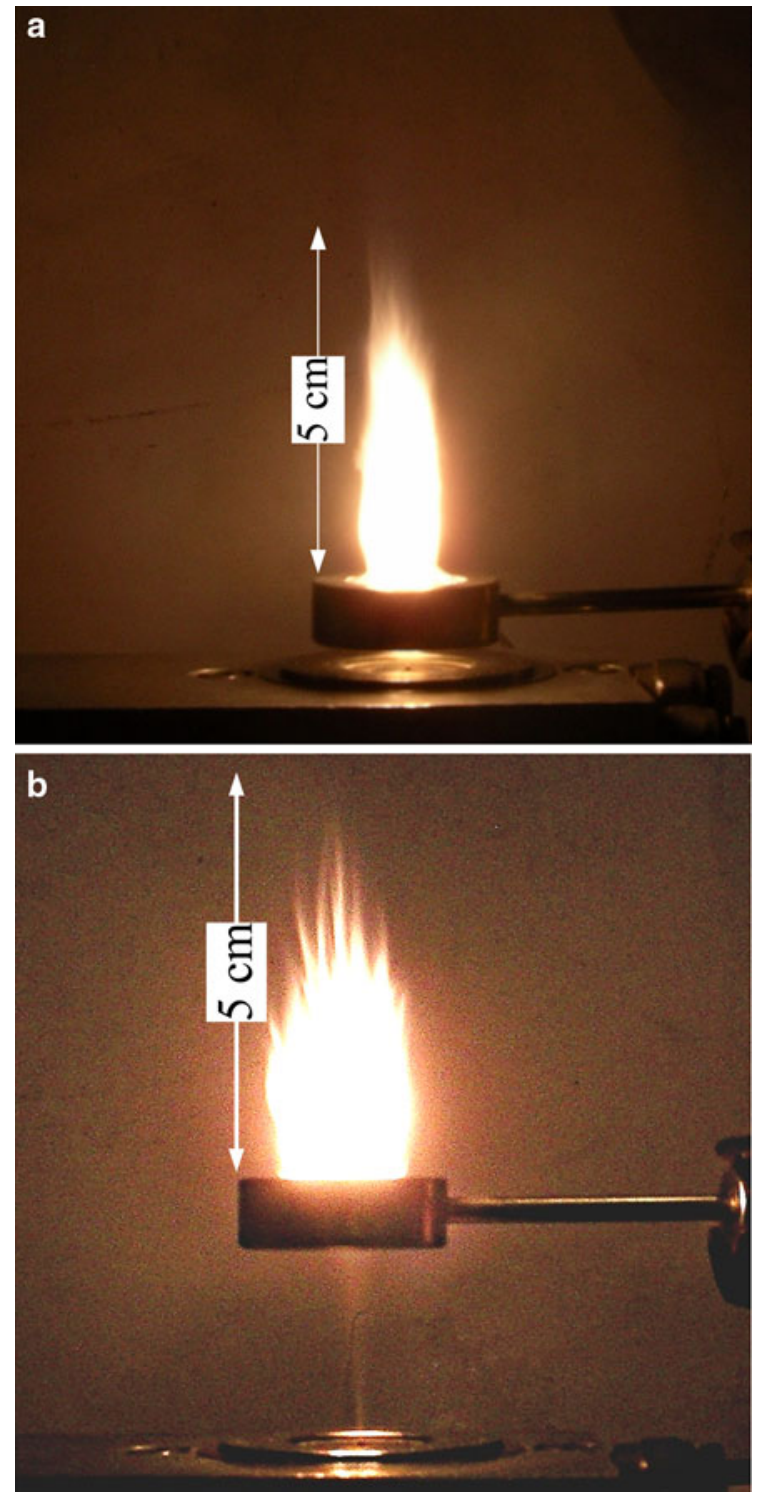

Fig. 2 Images of the FASP unit forming $\mathrm{Bi}_{2} \mathrm{O}_{3}$ nanoparticles with the ring issuing $2.5 \mathrm{~L} / \mathrm{min} \mathrm{C}_{2} \mathrm{H}_{2}$ at HAN of $\mathbf{a} 1$ and b $3 \mathrm{~cm}$. In both cases the spray jet of bismuth nitrate in ethanol/nitric acid solution is seen below the $\mathrm{C}_{2} \mathrm{H}_{2}$ ring that results in stable spray combustion. The bright yellow flame is wider and shorter at larger HAN as the spray droplets disperse and their velocity decreases

\section{Effect of fuel flow rate}

Figure 3 shows the XRD spectra of $\beta-\mathrm{Bi}_{2} \mathrm{O}_{3}$ made with 1-2.5 L/min of $\mathrm{C}_{2} \mathrm{H}_{2}$ or $1.5 / 2.5 \mathrm{~L} / \mathrm{min} \mathrm{CH}_{4} / \mathrm{O}_{2}$ with the FASP ring at $\mathrm{HAN}=1 \mathrm{~cm}$ (solid lines). A spectrum of FSP-made $\mathrm{Bi}_{2} \mathrm{O}_{3}$ (dashed line) with 1.5/ $2.5 \mathrm{~L} / \mathrm{min} \mathrm{CH}_{4} / \mathrm{O}_{2}$ is shown for comparison. Spectra 


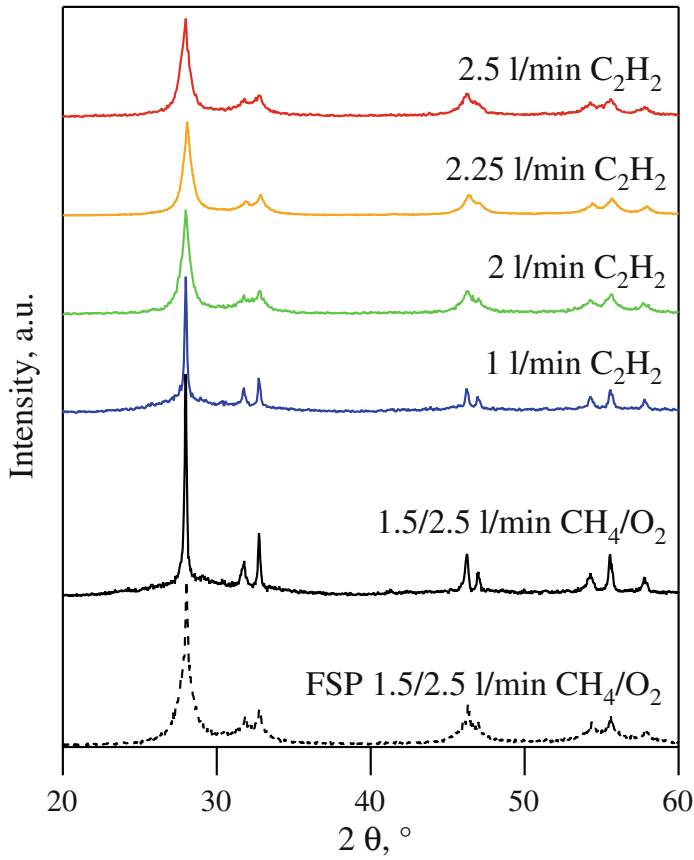

Fig. 3 XRD spectra of $\mathrm{Bi}_{2} \mathrm{O}_{3}$ produced by FSP (dashed line) and by FASP (solid lines) with the ignition ring at HAN $=1 \mathrm{~cm}$ without shielding tube and either $1-2.5 \mathrm{~L} / \mathrm{min}$ $\mathrm{C}_{2} \mathrm{H}_{2}$ or $1.5 / 2.5 \mathrm{~L} / \mathrm{min}$ premixed $\mathrm{CH}_{4} / \mathrm{O}_{2}$ flow through the ring. Powders made at low $\mathrm{C}_{2} \mathrm{H}_{2}$ flow rates and $\mathrm{CH}_{4} / \mathrm{O}_{2}$ show sharp peaks on top of humps indicating the presence of large crystals in the nanopowder. Increasing the flame enthalpy density by increasing the $\mathrm{C}_{2} \mathrm{H}_{2}$ flow rate resulted in more uniform powders, as indicated by broad $\mathrm{Bi}_{2} \mathrm{O}_{3}$ crystalline peaks $(2.25$ and $2.5 \mathrm{~L} / \mathrm{min} \mathrm{C}_{2} \mathrm{H}_{2}$ )

of powders made at low $\mathrm{C}_{2} \mathrm{H}_{2}$ flow rates $(<2 \mathrm{~L} / \mathrm{min})$ or premixed $\mathrm{CH}_{4} / \mathrm{O}_{2}$ are similar to the FSP-made ones (Madler and Pratsinis 2002). They exhibit sharp XRD peaks on top of broad ones, indicative of large and small crystallites in the product powder. At higher acetylene flow rates ( $\geq 2 \mathrm{~L} / \mathrm{min}$ ), however, the intensity of the peaks decreases while their width increases, indicative of finer $\mathrm{Bi}_{2} \mathrm{O}_{3}$ crystals. Powders made at these flow rates resemble those of unimodal crystal size distributions (Madler and Pratsinis 2002) made by standard FSP of mostly acetic acid-based solutions.

Figure 4 shows average crystallite sizes obtained from XRD spectra (e.g., Fig. 3) along with the corresponding BET-equivalent particle diameters for $\mathrm{HAN}=1 \mathrm{~cm}$ as a function of $\mathrm{C}_{2} \mathrm{H}_{2}$ flow rate. At the bottom of Fig. 4 and for up to $2 \mathrm{~L} / \mathrm{min} \mathrm{C}_{2} \mathrm{H}_{2}$, the GOF values for the two-mode fit (crosses) are always much lower than the one-mode fit (diamonds) of the

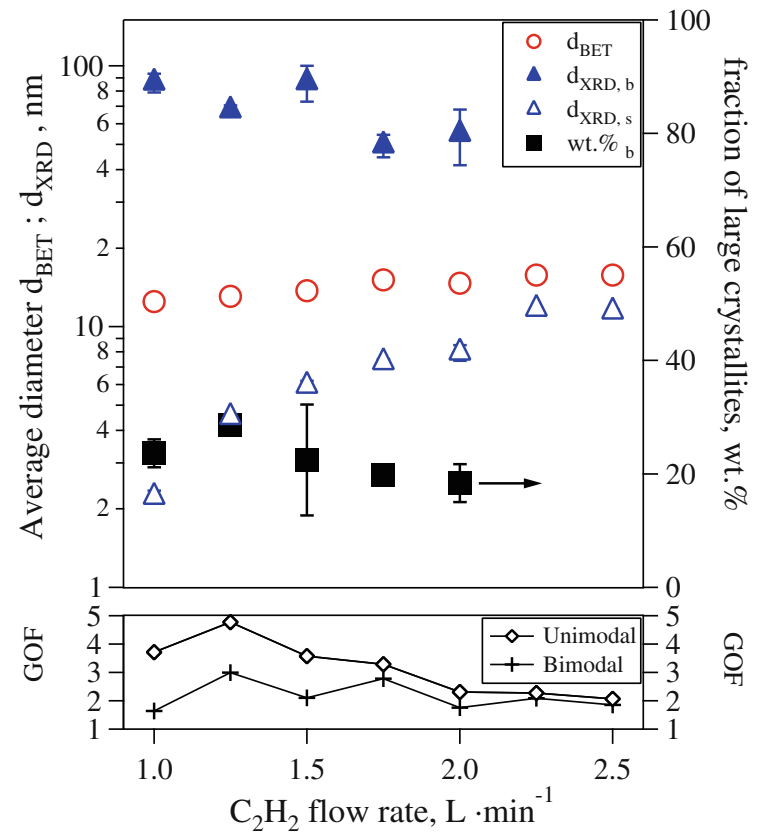

Fig. 4 XRD-crystallite sizes (triangles) and BET-equivalent particle diameters (circles) as a function of acetylene flow rate through the FASP ring at $\mathrm{HAN}=1 \mathrm{~cm}$. Small (open triangles) and large (filled triangles) crystallite sizes were fitted to the XRD spectra along with the mass fraction of the latter (squares). The Goodness of Fit (GOF, bottom diagram) is lower for the two-mode fit at low $\mathrm{C}_{2} \mathrm{H}_{2}$ flow rates indicating a better match to the spectra. For $\mathrm{C}_{2} \mathrm{H}_{2}$ flow rates $>2 \mathrm{~L} / \mathrm{min}$, the spectra are well described by a single crystallite mode indicating a more uniform product. Then, the two crystallite sizes converge toward a single one

XRD spectra. For instance, for $1 \mathrm{~L} / \mathrm{min}$ acetylene flow rate, the GOF with one and two modes are 3.7 and 1.6, respectively, indicating that the crystallinity of the product powder is described better by a broad distribution with large and small crystallites (Jossen et al. 2005b). The average large crystallite size (filled triangles) is $86 \mathrm{~nm}$ while the small one (open triangles) is $2.5 \mathrm{~nm}$. In Fig. 4, the two crystallite sizes of the two-mode fit are approaching each other for increasing $\mathrm{C}_{2} \mathrm{H}_{2}$ flow rates: at $2 \mathrm{~L} / \mathrm{min}$, the average large crystal size has decreased to $55 \mathrm{~nm}$ while that of the small crystals has increased to $8 \mathrm{~nm}$. Also the GOF-values for fitting one and two crystal sizes converge to 2.3 and 1.8 , respectively. The mass fraction of the large crystallite size (squares) is shown on the right ordinate of Fig. 4. For powders with bimodal fit, it decreases from about 30 to around 20 wt $\%$ with increased $\mathrm{C}_{2} \mathrm{H}_{2}$ flow. For $\mathrm{C}_{2} \mathrm{H}_{2}$ flow rates 
of $2.25 \mathrm{~L} / \mathrm{min}$ and above, the measured XRD-spectra could be described well by fitting only one crystallite mode. The GOF-values for two- and one-mode fit have almost the same value of 2.1 and 2.3, respectively, indicating the formation of a uniform powder as it appears to have a unimodal crystal size distribution anyway (Fig. 3). Seo et al. (2003) found also more uniform nanoparticulate products when increasing the flame temperature by increased propane flow. They concluded that the flame temperature is a key parameter for the production of a uniform product.

The $\mathrm{d}_{\mathrm{BET}}$ (circles) is increasing from 12.5 to $15.8 \mathrm{~nm}$ with increasing $\mathrm{C}_{2} \mathrm{H}_{2}$ flow rate from 1 to $2.5 \mathrm{~L} / \mathrm{min}$. The slightly larger BET-diameters at higher acetylene flow rates are attributed to increased high temperature particle residence times that lead to enhanced coalescence and sintering reducing the specific surface area (Pratsinis 1998). The $d_{B E T}$ is an integral property of the entire particle size distribution, so it does not give an accurate picture for broad or bimodal size distributions (Jossen et al. 2005a). When, however, unimodal $\mathrm{Bi}_{2} \mathrm{O}_{3}$ crystal size distributions are made at high $\mathrm{C}_{2} \mathrm{H}_{2}$ flow rates, the BET diameter is consistent with the XRD size indicating monocrystalline nanoparticles.

Figure 5 shows TEM-images of $\mathrm{Bi}_{2} \mathrm{O}_{3}$ formed at (a-c) 1 and (d) $2.5 \mathrm{~L} / \mathrm{min}$ acetylene flow and $\mathrm{HAN}=1 \mathrm{~cm}$. Large spherical particles of a few hundred $\mathrm{nm}$ in diameter along with nanosized particles of about $10 \mathrm{~nm}$ are observed at the low $\mathrm{C}_{2} \mathrm{H}_{2}$ flow rate (Fig. 5a, b). The electron diffraction pattern of a zone with a large particle (Fig. 5a, inset) shows bright diffraction spots that indicate large crystallites along with the diffuse diffraction halo rings indicating very small ones, thus supporting the XRD analysis (Figs. 3, 4). A HRTEM image (Fig. 5c) of the fine particles shown in Fig. 5a reveals crystallites of about $10 \mathrm{~nm}$ with well discernible lattice planes in agreement with the small crystallite
Fig. 5 Transmission electron microscopy images of powders made by FASP of Bi-nitrate with the ring delivering $1 \mathrm{~L} / \mathrm{min}(\mathbf{a}-\mathbf{c})$ and $2.5 \mathrm{~L} / \mathrm{min}$ (d) acetylene at $\mathrm{HAN}=1 \mathrm{~cm}$. Large spherical particles of few hundred nm co-exist with nanoparticles made at $1 \mathrm{~L} / \mathrm{min}(\mathbf{a}, \mathbf{b})$. The electron diffraction pattern of a zone with a spherical particle (inset a) shows bright reflection spots that indicate large crystallites along with diffuse diffraction halo rings indicating very small ones. The crystalline nature of the small particles shown in $\mathbf{a}$ is revealed by HRTEM c and electron diffraction (inset $\mathbf{c}$ ). A more uniform fine powder is made with $2.5 \mathrm{~L} / \mathrm{min} \mathrm{C}_{2} \mathrm{H}_{2}$ (d)
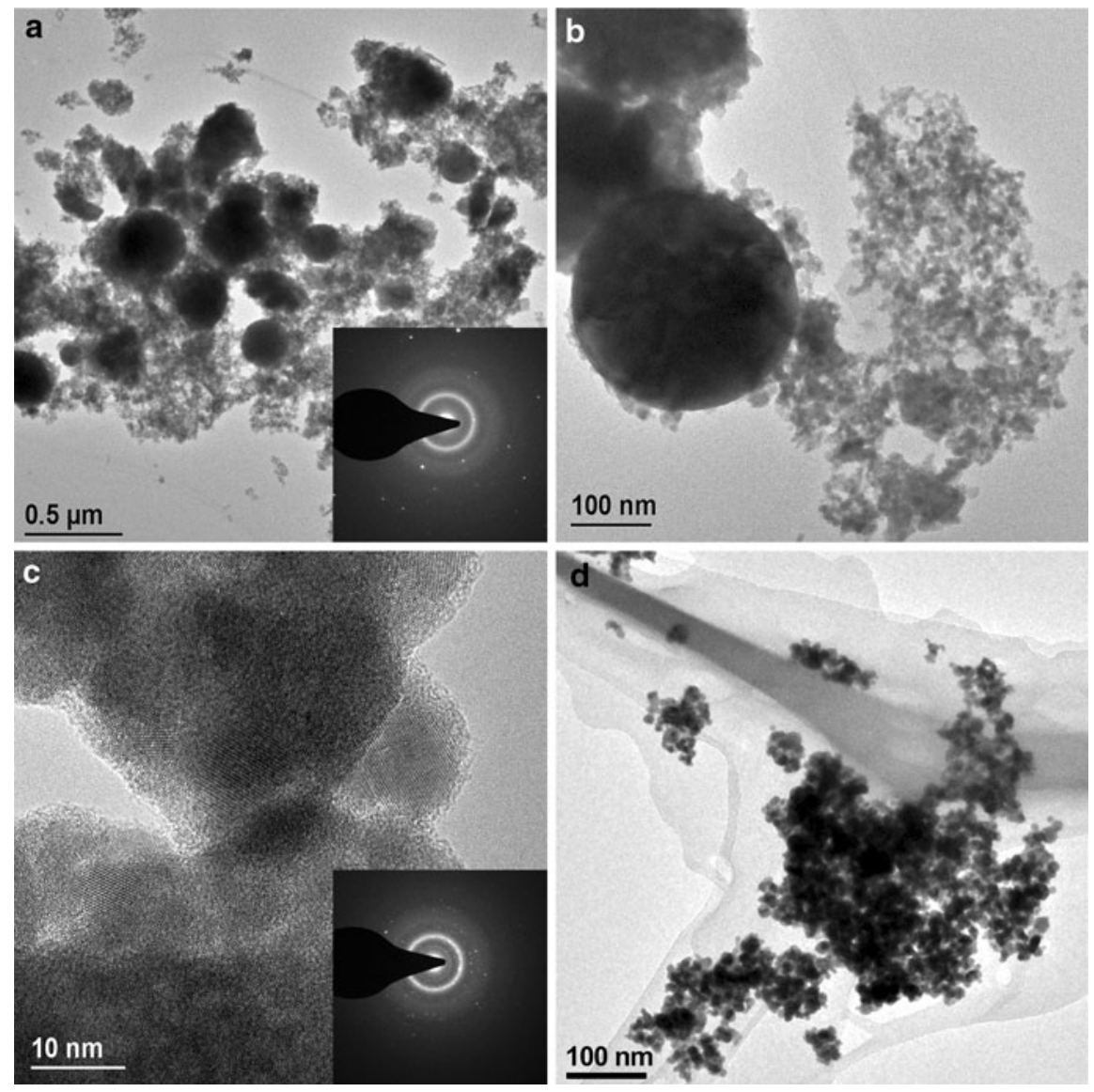
size mode seen in XRD. The crystalline nature of the fine particles is further proven by the corresponding local area diffraction pattern (Fig. 5c, inset). At $2.5 \mathrm{~L} / \mathrm{min}$ acetylene flow, a more uniform product is obtained (Fig. 5d), even though a few large particles are present as well (not shown).

The non-uniformity of the powder made at low acetylene flow rates can be explained by different particle formation routes (Madler et al. 2002b): The nanosized fraction is formed by evaporation of the precursor, nucleation in the gas phase and subsequent particle growth (gas-to-particle conversion). The large particles are formed by precursor precipitation inside the droplet and reaction (droplet-to-particle conversion). This occurs when the solvent is evaporating, leading to supersaturation and reaction of the precursor on the droplet surface or inside the droplet. Formation of a concentration gradient and surface precipitation on the droplets leads to hollow particles (Messing et al. 1993) and subsequent densification may lead to the observed dense large particle fraction (Tani et al. 2003, 2004).

Whenever a considerable amount of large particles was observed along with much smaller ones by electron microscopy, the XRD patterns (Fig. 3) showed narrow peaks on top of broad ones that could be better fitted (smaller GOF) by two modes for large and small crystallite sizes rather than one (Fig. 4). These two modes can be traced to particle formation by gas-to-particle and droplet-to-particle conversion (Madler et al. 2002b). So, GOF analysis of XRD spectra facilitates identification of crystallites having a unimodal or bimodal size distribution.

The enthalpy content of the flame influences the high temperature particle residence time and subsequently the product homogeneity. Here the increase in flame enthalpy by increasing the $\mathrm{C}_{2} \mathrm{H}_{2}$ flow rate reduced the fraction of large particles leading to formation of more homogeneous products (Jossen et al. 2005b). With increasing $\mathrm{C}_{2} \mathrm{H}_{2}$ flow through the ring, the maximum flame temperature and high temperature particle residence time are increased. This enhances evaporation of all droplet components, so less precursor precipitates within the droplet (surface or inside), reducing the fraction of large particles. Another possible mechanism increasing powder uniformity is that fracture of hollow, shelllike particles is promoted in the high temperature acetylene flame by internal pressure of the heated solvent (Gardner and Messing 1984). This might reduce the amount of large solid particles which are generated by densification of hollow ones. Eventually the evaporation of the precipitated material of the hollow particles is also promoted by the high temperature of the acetylene flame. This might be possible for $\mathrm{Bi}_{2} \mathrm{O}_{3}$ having a relatively low $\left(1890{ }^{\circ} \mathrm{C}\right)$ boiling point (Lide 2007).

Figure 6 shows the cumulative mass- and frequency agglomerate $\mathrm{Bi}_{2} \mathrm{O}_{3}$ particle size distributions by X-ray disc centrifuge made at 1.0 (blue broken line), 1.5 (green dotted line), 2.0 (orange dot-broken line), and 2.5 (red solid line) $\mathrm{L} / \mathrm{min}_{2} \mathrm{H}_{2}$. The size distribution of the powder made at the lowest $\mathrm{C}_{2} \mathrm{H}_{2}$ flow rate shows about $55 \mathrm{wt} \%$ large particles $(>100 \mathrm{~nm})$. Increasing the $\mathrm{C}_{2} \mathrm{H}_{2}$ flow rate reduces this fraction to $20 \%(1.5 \mathrm{~L} /$ $\min$ ) and $15 \%$ (2 and $2.5 \mathrm{~L} / \mathrm{min})$. Particle sizes measured by X-ray disc centrifuge are bigger than those obtained from TEM or nitrogen adsorption $\left(\mathrm{d}_{\mathrm{BET}}\right)$ as the hydrodynamic particle diameters measured by X-ray disc centrifuge correspond best to agglomerate or aggregate sizes rather than to primary particle sizes (Schulz et al. 2008). Despite this and the fact that product agglomerates might not be fully disintegrated, the large fraction of $>100 \mathrm{~nm}$ particles in powders made at low acetylene flow rates indicates that the droplet-to-particle conversion pathway is more pronounced at these conditions, further supporting the reasoning from TEM and XRD analyses.

Effect of ignition flame location and air entrainment

Figure 7 shows the effect of ignition height (HAN) on size and crystallinity of product particles made by FASP at high $(2.5 \mathrm{~L} / \mathrm{min})$ acetylene flow rate. The BET-equivalent particle size (circles) is rather constant at about $15 \mathrm{~nm}$ for the investigated HAN = $0.65-3 \mathrm{~cm}$. For HAN up to $1.25 \mathrm{~cm}$ only one crystallite size (open triangles) describes well the measured XRD spectra indicative of a unimodal crystallite size distribution as seen already in Figs. 3 and 4. With increasing HAN up to $1.25 \mathrm{~cm}$, both product primary particle and crystallite sizes increase. An increase of HAN gives more time for droplet dispersion and evaporation, facilitating particle formation by gas-to-particle conversion.

At $\mathrm{HAN} \geq 1.5 \mathrm{~cm}$, however, the XRD spectra are described best by a bimodal crystallite size 


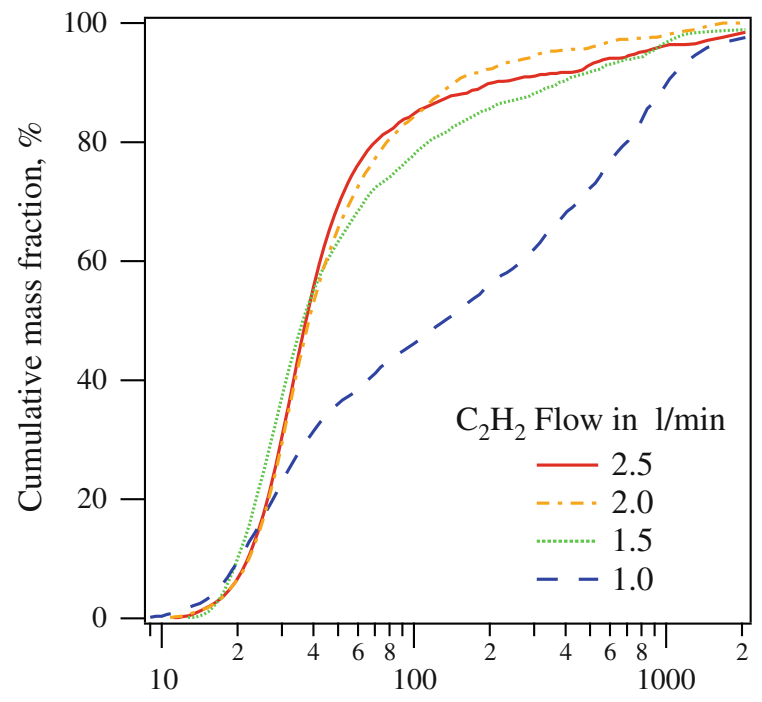

Agglomerate size, $\mathrm{nm}$

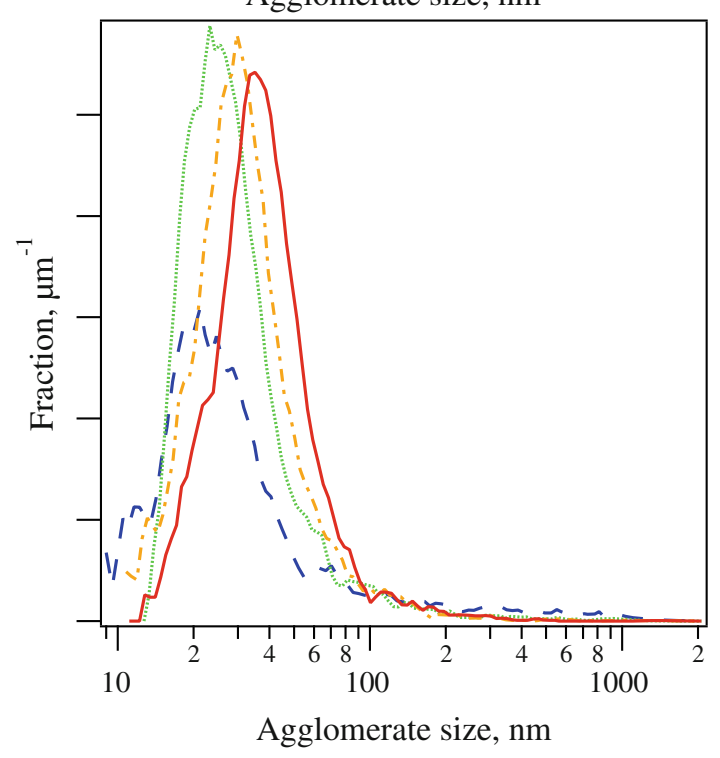

Fig. 6 Cumulative and frequency aggregate or agglomerate $\mathrm{Bi}_{2} \mathrm{O}_{3}$ particle mass size distributions by $\mathrm{X}$-ray disc centrifuge of the powders produced at $\mathrm{HAN}=1 \mathrm{~cm}$ and different $\mathrm{C}_{2} \mathrm{H}_{2}$ flow rates. At low $\mathrm{C}_{2} \mathrm{H}_{2}$ flows (e.g., $1 \mathrm{~L} / \mathrm{min}$ ) larger fractions of particles $>100 \mathrm{~nm}$ are obtained consistent with TEM and XRD analyses

distribution as seen by the GOF diagram at the bottom of Fig. 7. At HAN $=1.5 \mathrm{~cm}$ note the large error associated with both crystallite sizes (triangles) and mass fraction of large crystallites (squares) indicative of the ambiguity of deciphering between the two sizes by XRD. Beyond this HAN, however, the bimodality of the crystallite size distributions is undisputable given the difference in GOF between

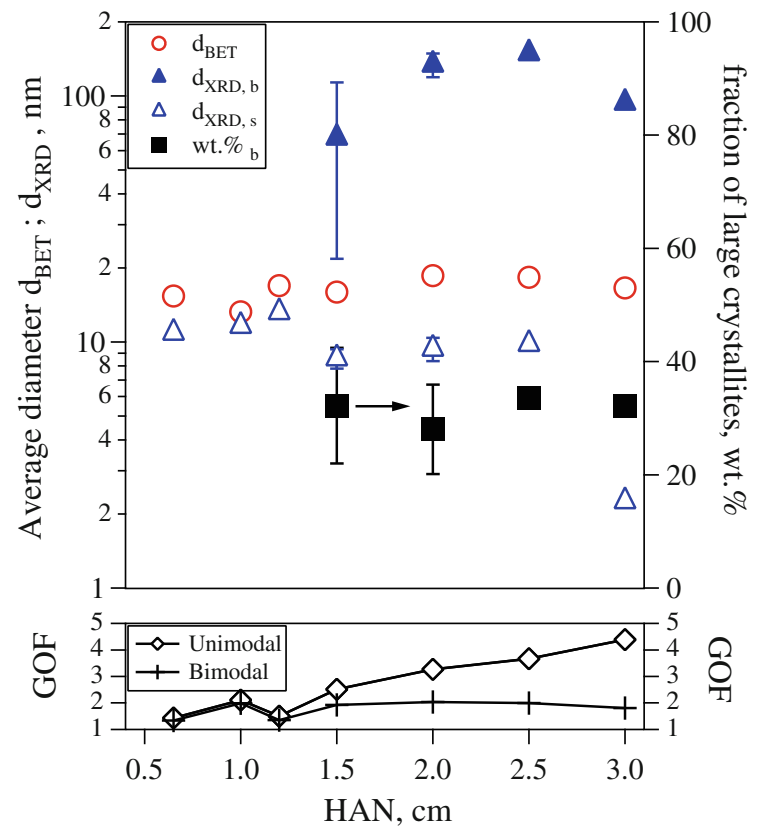

Fig. 7 XRD-crystallite and primary particle diameters of $\mathrm{Bi}_{2} \mathrm{O}_{3}$ made with $2.5 \mathrm{~L} / \mathrm{min}$ acetylene flow through the FASP ring as a function of HAN and without shielding tube around the spray. At distances larger than $1.25 \mathrm{~cm}$ formation of nonuniform $\mathrm{Bi}_{2} \mathrm{O}_{3}$ takes place as the GOF (bottom) for a bimodal XRD fit (crosses) is smaller than for a unimodal one (diamonds). This is attributed to entrainment of surrounding air into the spray jet and reduction of flame enthalpy density and temperature

unimodal and bimodal fit of the XRD spectra at the bottom of Fig. 7. The average size of large crystallites ranges from $68 \mathrm{~nm}$ at $\mathrm{HAN}=1.5 \mathrm{~cm}$ to $94 \mathrm{~nm}$ at $\mathrm{HAN}=3 \mathrm{~cm}$, while that of small crystallites is 9.4 and $2.5 \mathrm{~nm}$, respectively. For bimodal powders, the mass fraction of the large crystallites is about $30 \mathrm{wt} \%$ at all HAN. When a bimodal crystallite size distribution forms, the large crystallite size increases up to $\mathrm{HAN}=2.5 \mathrm{~cm}$ and then decreases as complete spray combustion does not take place with the FASP ring at higher HAN. At the same time, the small crystallite size remains rather constant until HAN $=2.5 \mathrm{~cm}$ and then it decreases also. Above HAN $=3 \mathrm{~cm}$, however, the powders were rather sticky indicating that incomplete combustion took place since the flame temperature had been substantially reduced.

The formation of non-uniform products for HAN $\geq 1.5 \mathrm{~cm}$ can be explained by dilution of the spray by air entrainment prior to ignition, lowering the flame temperature and enthalpy density. Heine 
et al. (2006) showed how the entrainment of surrounding air has a strong effect on particle size as it reduces the flame temperature by mixing surrounding cold air with the hot flame jet. When increasing the ring's HAN, the precursor spray jet has been expanded or widened (Fig. 1b) and diluted by mixing with surrounding air. The air entrainment increases for larger HAN and thus the resulting high temperature particle residence time and flame enthalpy density decrease. Note that with increasing HAN, the GOF for unimodal fit of XRD spectra increases, indicating that such a representation of the spectra is less accurate since the bimodality of the crystallite distribution increases (bottom of Fig. 7) and the corresponding GOF is rather constant.

Figure 8 shows the BET-equivalent diameters (circles) and XRD crystallite sizes (triangles) for $2.5 \mathrm{~L} / \mathrm{min}$ of $\mathrm{C}_{2} \mathrm{H}_{2}$ as a function of HAN as in Fig. 7 but now the air entrainment below the FASP ring is prevented by placing a shielding tube between the spray nozzle and the FASP ring (Fig. 1). The BETequivalent particle diameter increases from about 15 to $19.5 \mathrm{~nm}$ when increasing HAN from 1 to $3 \mathrm{~cm}$. For these HAN, the crystallite size grows from 12 to $20.5 \mathrm{~nm}$ that is similar to $\mathrm{d}_{\mathrm{BET}}$, indicating monocrystalline particles. The GOF values for unimodal and bimodal XRD fits are practically indistinguishable indicating formation of uniform product powder at all HAN. When air entrainment below the FASP ring is prevented, higher spray flame temperatures are attained as less gas has to be heated by the $\mathrm{C}_{2} \mathrm{H}_{2}-$ $\mathrm{O}_{2}$ flame, so droplets evaporate more efficiently before mixing with air entrained further downstream of the FASP ring. As a result, gas-to-particle conversion is favored and droplet-to-particle conversion is suppressed so a largely uniform product is formed. The insets in Fig. 8 show TEM images of $\mathrm{Bi}_{2} \mathrm{O}_{3}$ produced at $\mathrm{HAN}=1$ (left top) and $3 \mathrm{~cm}$ (right bottom). Observed particle diameters correspond well with the measured primary particle and crystallite sizes.

\section{Conclusions}

A new flame reactor design (flame assisted spray pyrolysis, FASP configuration) was presented decoupling the flame enthalpy content from the precursor solution chemistry. The new reactor was compared to

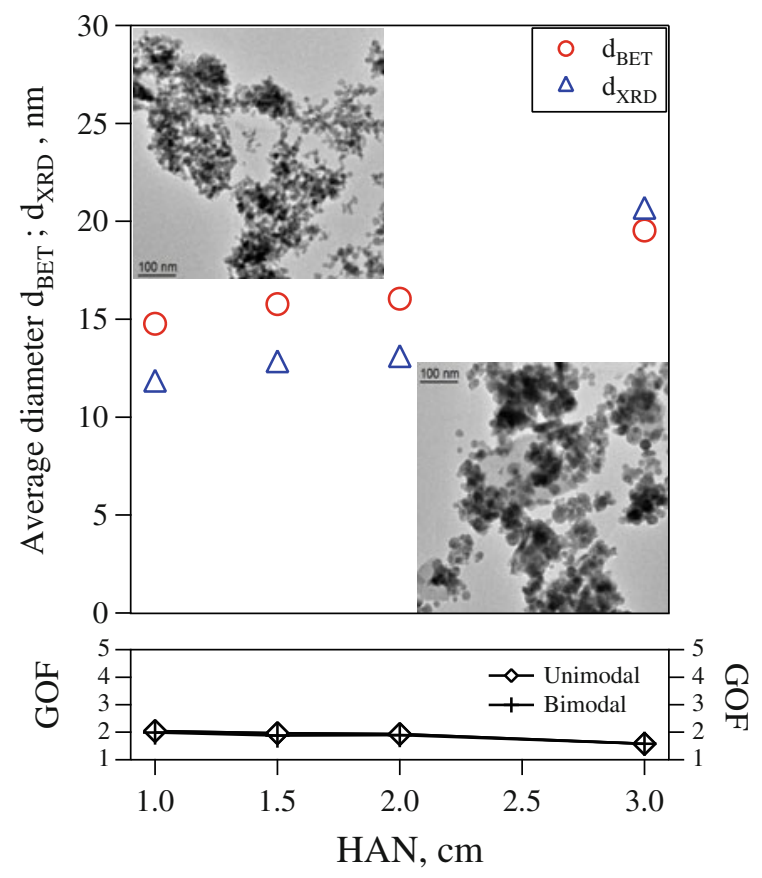

Fig. 8 XRD crystallite and primary particle diameters of powders made at $2.5 \mathrm{~L} / \mathrm{min} \mathrm{C}_{2} \mathrm{H}_{2}$ and $\mathrm{HAN}=1-3 \mathrm{~cm}$ with the optional shielding tube preventing air entrainment in the non-burning spray (Fig. 1). As the GOF values for bi- and unimodal XRD fit are indistinguishable, homogeneous product powders are obtained at all HAN. The primary particle diameter and the average crystallite size increase with increasing HAN as this reduces heat loss for heating entrained air allowing droplets enough residence time to evaporate and mix with the dispersion $\mathrm{O}_{2}$ prior to mixing with surrounding air. Insets show TEM images of $\mathrm{Bi}_{2} \mathrm{O}_{3}$ produced at $\mathrm{HAN}=1$ (left) and $3 \mathrm{~cm}$ (right) consistent with GOF values (below) for unimodal nanoparticles

the standard flame spray pyrolysis (FSP) configuration (Madler and Pratsinis 2002) for bismuth oxide synthesis from bismuth nitrate solutions that formed non-uniform bimodal powders by conventional FSP. The dependence of product powder uniformity on spray flame configuration was investigated for a low enthalpy containing precursor-ethanol solution. A clear trend to formation of more homogeneous and monomodal bismuth nanoparticles could be observed for increased enthalpy density by increasing the fuel gas (acetylene) flow in FASP.

Ignition at larger HAN allowed the precursor solution to evaporate from spray droplets and mix with the supplied oxygen to form a rather premixed flame. Increasing of the HAN promoted air entrainment to the spray before ignition that reduced the 
combustion enthalpy density of the spray flame and resulted in undesired formation of bimodal product. When preventing air entrainment by enclosure of the spray prior to ignition, the formation of bimodal product powders was prevented.

The addition of enthalpy through the gas phase might allow selection of inexpensive metal-nitrate and other inorganic precursors and ethanol-like solvents for industrial scale production of nanoparticles, as it has potential to save costs compared to elaborate and costly oxygenated precursor mixtures.

Acknowledgment The support of Dr. F. Krumeich for TEM is gratefully acknowledged, as well as financial support by ETH Research Grant TH-23 06-3 and European Research Council.

\section{References}

Bickmore CR, Waldner KF, Treadwell DR, Laine RM (1996) Ultrafine spinel powders by flame spray pyrolysis of a magnesium aluminum double alkoxide. J Am Ceram Soc 79(5):1419-1423

Blower SK, Greaves C (1988) The structure of beta- $\mathrm{Bi}_{2} \mathrm{O}_{3}$ from powder neutron-diffraction data. Acta Crystallogr $\mathrm{C}$ 44:587-589

Cheary RW, Coelho A (1992) A fundamental parameters approach to X-ray line-profile fitting. J Appl Crystallogr 25:109-121

Gardner TJ, Messing GL (1984) Magnesium salt decomposition and morphological development during evaporative decomposition of solutions. Thermochim Acta 78(1-3):17-27

Heine MC, Madler L, Jossen R, Pratsinis SE (2006) Direct measurement of entrainment during nanoparticle synthesis in spray flames. Combust Flame 144(4):809-820

Jossen R, Mueller R, Pratsinis SE, Watson M, Akhtar MK (2005a) Morphology and composition of spray-flamemade yttria-stabilized zirconia nanoparticles. Nanotechnology 16(7):S609-S617

Jossen R, Pratsinis SE, Stark WJ, Madler L (2005b) Criteria for flame-spray synthesis of hollow, shell-like, or inhomogeneous oxides. J Am Ceram Soc 88(6):1388-1393

Kilian A, Morse TF (2001) A novel aerosol combustion process for the high rate formation of nanoscale oxide particles. Aerosol Sci Technol 34(2):227-235

Kriegel R, Topfer J, Preuss N, Grimm S, Boer J (1994) Flame pyrolysis - a preparation route for ultrafine powders of metastable beta-SrMnO 3 and $\mathrm{NiMn}_{2} \mathrm{O}_{4}$. J Mater Sci Lett 13(15):1111-1113

Lewis DJ (1991) Technique for producing mullite and other mixed-oxide systems. J Am Ceram Soc 74(10):2410-2413

Lide DR (2007) Properties of the elements and inorganic compounds. In: Lide DR (ed) CRC handbook of chemistry and physics: a ready-reference book of chemical and physical data, 88 edn. CRC, Boca Raton
Loher S, Stark WJ, Maciejewski M, Baiker A, Pratsinis SE, Reichardt D, Maspero F, Krumeich F, Gunther D (2005) Fluoro-apatite and calcium phosphate nanoparticles by flame synthesis. Chem Mater 17(1):36-42. doi:10.1021/ $\mathrm{cm} 048776 \mathrm{c}$

Madler L (2004) Liquid-fed aerosol reactors for one-step synthesis of nano-structured particles. Kona 22:107-120

Madler L, Pratsinis SE (2002) Bismuth oxide nanoparticles by flame spray pyrolysis. J Am Ceram Soc 85(7):1713-1718

Madler L, Kammler HK, Mueller R, Pratsinis SE (2002a) Controlled synthesis of nanostructured particles by flame spray pyrolysis. J Aerosol Sci 33(2):369-389

Madler L, Stark WJ, Pratsinis SE (2002b) Flame-made ceria nanoparticles. J Mater Res 17(6):1356-1362

Marshall BS, Telford I, Wood R (1971) Field method for determination of zinc oxide fume in air. Analyst 96(1145): 569-578

Matsoukas T, Friedlander SK (1991) Dynamics of aerosol agglomerate formation. J Colloid Interf Sci 146(2): 495-506

McCusker LB, Von Dreele RB, Cox DE, Louer D, Scardi P (1999) Rietveld refinement guidelines. J Appl Crystallogr 32:36-50

Merkle BD, Kniseley RN, Schmidt FA, Anderson IE (1990) Superconducting $\mathrm{YBa}_{2} \mathrm{Cu}_{3} \mathrm{O}_{\mathrm{x}}$ particulate produced by total consumption burner processing. Mater Sci Eng A-Struct 124(1):31-38

Messing GL, Zhang SC, Jayanthi GV (1993) Ceramic powder synthesis by spray-pyrolysis. J Am Ceram Soc 76(11): 2707-2726

Mueller R, Madler L, Pratsinis SE (2003) Nanoparticle synthesis at high production rates by flame spray pyrolysis. Chem Eng Sci 58(10):1969-1976

Pratsinis SE (1998) Flame aerosol synthesis of ceramic powders. Prog Energy Combust Sci 24(3):197-219

Purwanto A, Wang WN, Ogi T, Lenggoro IW, Tanabe E, Okuyama K (2008) High luminance YAG: Ce nanoparticles fabricated from urea added aqueous precursor by flame process. J Alloy Compd 463(1-2):350-357. doi: 10.1016/j.jallcom.2007.09.023

Rohner F, Ernst FO, Arnold M, Hibe M, Biebinger R, Ehrensperger F, Pratsinis SE, Langhans W, Hurrell RF, Zimmermann MB (2007) Synthesis, characterization, and bioavailability in rats of ferric phosphate nanoparticles. J Nutr 137(3):614-619

Sahm T, Madler L, Gurlo A, Barsan N, Pratsinis SE, Weimar U (2004) Flame spray synthesis of tin dioxide nanoparticles for gas sensing. Sens Actuators B-Chem 98(2-3):148-153. doi:10.1016/j.snb.2003.10.003

Schulz H, Schimmoeller B, Pratsinis SE, Salz U, Bock T (2008) Radiopaque dental adhesives: dispersion of flamemade $\mathrm{Ta}_{2} \mathrm{O}_{5} / \mathrm{SiO}_{2}$ nanoparticles in methacrylic matrices. J Dent 36(8):579-587

Seo DJ, Park SB, Kang YC, Choy KL (2003) Formation of $\mathrm{ZnO}, \mathrm{MgO}$ and $\mathrm{NiO}$ nanoparticles from aqueous droplets in flame reactor. J Nanopart Res 5(3-4):199-210

Seo DJ, Ryu KO, Park SB, Kim KY, Song RH (2006) Synthesis and properties of $\mathrm{Ce}_{1-\mathrm{x}} \mathrm{Gd}_{\mathrm{x}} \mathrm{O}_{2-\mathrm{x} / 2}$ solid solution prepared by flame spray pyrolysis. Mater Res Bull 41(2):359-366. doi:10.1016/j.materresbull.2005.08.012 
Stark WJ, Madler L, Maciejewski M, Pratsinis SE, Baiker A (2003) Flame synthesis of nanocrystalline ceria-zirconia: effect of carrier liquid. Chem Commun (5):588-589

Strobel R, Pratsinis SE (2007) Flame aerosol synthesis of smart nanostructured materials. J Mater Chem 17:4743-4756

Strobel R, Baiker A, Pratsinis SE (2006) Aerosol flame synthesis of catalysts. Adv Powder Technol 17(5):457-480

Tani T, Watanabe N, Takatori K, Pratsinis SE (2003) Morphology of oxide particles made by the emulsion combustion method. J Am Ceram Soc 86(6):898-904
Tani T, Takatori K, Pratsinis SE (2004) Dynamics of hollow and solid alumina particle formation in spray flames. J Am Ceram Soc 87(3):523-525

Tikkanen J, Gross KA, Berndt CC, Pitkanen V, Keskinen J, Raghu S, Rajala M, Karthikeyan J (1997) Characteristics of the liquid flame spray process. Surf Coat Technol $90(3): 210-216$ 